\title{
EXAMPLES OF DIRECT PRODUCTS OF SEMIGROUPS OR GROUPOIDS ${ }^{1}$
}

\author{
BY TAKAYUKI TAMURA
}

\author{
Communicated by George E. Forsythe, December 31, 1962
}

1. Introduction. The direct product $G_{1} \times \cdots \times G_{n}$ of groupoids $G_{i}$ 's is defined by $G_{1} \times \cdots \times G_{n}=\left\{\left(x_{1}, \cdots, x_{n}\right) ; x_{i} \in G_{i}, i=1, \cdots, n\right\}$ where $\left(x_{1}, \cdots, x_{n}\right)=\left(y_{1}, \cdots, y_{n}\right)$ means $x_{i}=y_{i}(i=1, \cdots, n)$ and $\left(x_{i}, \cdots, x_{n}\right)\left(y_{1}, \cdots, y_{n}\right)=\left(x_{1} y_{1}, \cdots, x_{n} y_{n}\right)$.

If a semigroup $A$ contains a subsemigroup which is isomorphic onto $B$, we say that $A$ contains $B$.

Let $i_{t}$ be one of $1, \cdots, n$, and let $i_{t} \neq i_{s}$ if $t \neq s . G_{i_{1}} \times \cdots \times G_{i_{m}}$, $1 \leqq m<n$, is called a partial product with length $m$ of $G_{1} \times \cdots \times G_{n}$.

It is familiar that if $G_{i}$ 's are groups, their direct product contains every partial product; but this is not true in the case of groupoids, not even in the case of semigroups. We can show the examples of direct product which contain no partial product. Such a direct product is called a completely exclusive direct product.

THEOREM 1. $G_{1} \times \cdots \times G_{n}$ is a completely exclusive direct product of groupoids $G_{1}, \cdots, G_{n}$ if and only if no partial product with length $n-1, G_{1} \times \cdots \times G_{i-1} \times G_{i+1} \times \cdots \times G_{n}$ is homomorphic into $G_{i}$ $(i=1, \cdots, n)$.

Corollary 1. If $G_{1} \times \cdots \times G_{n}$ is completely exclusive, then a partial product with length $>1$ is also completely exclusive.

2. Example for groupoids. Let $G_{n}$ be a set of $n$ elements and $\phi$ be a cycle of the $n$ elements, i.e., a cyclic permutation. The product of elements of $G_{n}$ is defined by:

$$
a b=(a) \phi \quad \text { for all } a, b \in G_{n} .
$$

Such a groupoid $G_{n}$ is called a cyclic left constant groupoid. A cyclic left constant groupoid is uniquely determined by $n$ within isomorphism, and we see that a cyclic left constant groupoid has neither idempotent element nor proper subgroupoid, and that if $m_{1}, \cdots, m_{k}$ are relatively prime in pairs and if $G_{m_{1}}, \cdots, G_{m_{k}}$ are cyclic left constant groupoids of order $m_{1}, \cdots, m_{k}$ respectively, then $G_{m_{1}} \times \cdots$ $\times G_{m_{k}}$ is also a cyclic left constant groupoid.

1 This paper was delivered at the meeting of the American Mathematical Society, at Santa Barbara, California, November 18, 1961. See Notices Amer. Math. Soc. 8 (1961), 513. The precise proof will be given elsewhere. 
TheOREM 2. Let $p_{1}<p_{2}<\cdots<p_{n}$ be positive prime numbers. The direct product of cyclic left constant groupoids $G_{p_{i}}(i=1, \cdots, n)$ is completely exclusive.

3. Example for commutative semigroups. Let $S_{1}$ be the additive semigroup of all positve rational numbers, $S_{2}$ be the multiplicative semigroup of all rational numbers $>1$. Then we have an example of completely exclusive direct product:

$$
S_{1} \times S_{2} .
$$

For this purpose we use the properties of power-joined semigroups and naturally totally ordered archimedean commutative semigroups; and furthermore we can generalize the above example.

A semigroup $S$ is said to be power-joined if for any two elements $x$ and $y$ there are natural numbers $n$ and $m$ such that $x^{n}=y^{m}$. The property of power-joinedness is preserved by subsemigroups and homomorphisms, and we can prove that a power-joined subsemigroup of $S_{2}$ is isomorphic into the additive semigroup of all positive integers.

A naturally totally ordered archimedean commutative semigroup $S$ (abbreviated n.t.o.a.c. semigroup) is a naturally totally ordered commutative semigroup in the sense of [1] which satisfies the following additional conditions:

(1) $x>y$ implies $x z>y z$ for every $z \in S$.

(2) $x y>x$ for every $x, y \in S$.

(3) For any elements $x$ and $y$, there is a natural number $n$ such that $x^{n}>y$.

Then we can determine every proper homomorphism of $S$ by means of $[2 ; 3]$ and [4]. Consequently we have

THEOREM 3. A proper homomorphic image of a n.t.o.a.c. semigroup is a unipotent semigroup. Therefore, a n.t.a.o.c. semigroup cannot be properly homomorphic into any n.t.o.a.c. semigroup. We see that if n.t.o.a.c. semigroups $A$ and $B$ are not isomorphic into each other, the direct product $A \times B$ is completely exclusive.

4. Example for three semigroups. Let $F_{1}$ be a semigroup satisfying the following conditions:

(4) For any $a, b \in F_{1}$ there is $c \in F_{1}$ such that $a=b c$.

(5) $c \neq b c$ for every $b, c \in F_{1}$.

Clearly $F_{1}$ contains no idempotent and is not commutative. Let $F_{1}^{*}$ be the dual semigroup of $F_{1}$. Then we have

LEMMA 1. $F_{1} \times F_{1}^{*}$ is not homomorphic into a commutative semigroup without idempotent. 
Suppose that $F$ is a semigroup which satisfies not only (4), (5) but also (6), (7):

(6) Right cancellative: $f h=g h$ implies $f=g$.

(7) For any $f, g \in F$ there are elements $k, h \in F$ such that $k f=h g$.

Let $S$ be a n.t.o.a.c. semigroup.

Lemma 2. $F \times S$ is homomorphic into $F^{*}$ if and only if $S$ is isomorphic into $F$.

As the dual form of Lemma 2, we can say that $F^{*} \times S$ is homomorphic into $F$ if and only if $S$ is isomorphic into $F^{*}$.

By Lemmas 1, 2 and Theorem 1, we have

TheORem 4. $F \times S \times F^{*}$ is a completely exclusive direct product if and only if $S$ is not isomorphic into $F$.

5. Example of $F \times S \times F^{*}$. A concrete example of $F \times S \times F^{*}$ is given as follows: Consider two sequences of positive rational numbers

$$
\begin{aligned}
& 0=a_{0}<a_{1}<\cdots<a_{k-1}<a_{k}=1 \\
& 0=b_{0}<b_{1}<\cdots<b_{k-1}<b_{k}<1
\end{aligned}(k: \text { arbitrary } \geqq 1)
$$

such that, letting

$$
c_{i}=\frac{b_{i+1}-b_{i}}{a_{i+1}-a_{i}}, \quad c_{i} \neq c_{i+1}, \quad i=0,1, \cdots, k-1 .
$$

We define a system of linear functions $f_{i}(i=0,1, \cdots, k-1)$ on the interval $\left[a_{i}, a_{i+1}\right]$ as follows:

(8) $(x) f_{0}=c_{0} x, 0 \leqq x \leqq a_{1}$.

(9) $(x) f_{i}=c_{i}\left(x-a_{i}\right)+\left(a_{i}\right) f_{i-1}, a_{i} \leqq x \leqq a_{i+1}, i=1, \cdots, k$.

Joining $f_{0}, f_{1}, \cdots, f_{k}$ successively, we get a function $f$ on $[0,1]$. $F$ denotes the set of all functions $f$. Then $F$ is a semigroup with respect to the usual multiplication of functions, and $F$ satisfies (4), (5), (6) and (7). Let $S_{1}$ be the same semigroup as defined in $\$ 3$. Then we can prove that $S_{1}$ is not isomorphic into $F$. Thus we have an example of a completely exclusive direct product

$$
F \times S_{1} \times F^{*}
$$

by Theorem 4 .

6. Added remark. After writing this paper, we obtained an example of a completely exclusive direct product of an arbitrary finite number of commutative semigroups. 
Let $p$ be a positive prime number, and $T_{p}$ be the semigroup of all positive rational numbers of the form $j / p^{i}$ with respect to the usual addition. Let $p_{1}, p_{2}, \cdots, p_{n}$ be distinct positive prime numbers. Then $T_{p_{1}} \times \cdots \times T_{p_{n}}$ is a completely exclusive direct product.

\title{
REFERENCES
}

1. A. H. Clifford, Naturally totally ordered commutative semigroups, Amer. J. Math. 76 (1954), 631-646.

2. - Totally ordered commutative semigroups, Bull. Amer. Math. Soc. 64 (1958), 305-316.

3. O. Hölder, Die Axiome der Quantität und die Lehre vom Mass, Ber. Verh. Sächs. Ges. Wiss. Leipzig Math.-Phys. Kl. 53 (1901), 1-64.

4. T. Tamura, Commutative nonpotent archimedean semigroup with cancellation law. I, J. Gakugei Tokushima Univ. 8 (1957), 5-11.

University of California, Davis

\section{THE INDEX OF ELLIPTIC OPERATORS ON COMPACT MANIFOLDS}

\author{
BY M. F. ATIYAH AND I. M. SINGER ${ }^{1}$
}

Communicated by Raoul Bott, February 1, 1963

Introduction. In his paper [16] Gel'fand posed the general problem of investigating the relationship between topological and analytical invariants of elliptic differential operators. In particular he suggested that it should be possible to express the index of an elliptic operator (see $\$ 1$ for the definition) in topological terms. This problem has been taken up by Agranovic $[2 ; 3]$, Dynin $[3 ; 14 ; 15]$, Seeley $[20 ; 21]$ and Vol'pert [22] who have solved it in special cases. The purpose of this paper is to give a general formula for the index of an elliptic operator on any compact oriented differentiable manifold (Theorem 1). As a special case of this formula we get the Hirzebruch-RiemannRoch theorem for any compact complex manifold (Theorem 3). This was previously known only for projective algebraic manifolds. Some other special cases, of interest in differential topology, are discussed in $\$ 3$.

We are greatly indebted to A. P. Calderon, L. Nirenberg, and R. T. Seeley for their generous help.

1. Elliptic operators. Let $X$ be a compact oriented smooth mani-

${ }^{1}$ Part of this work was done with the first author supported by the National Science Foundation and the second author holding a Sloan Fellowship. 